\title{
DISCUSSION ON FLOW-THROUGH PHENOMENA IN THE AIR GAUGE CASCADE
}

\author{
Czesław Janusz JERMAK ${ }^{*}$ \\ "Division of Metrology and Measurement Systems, Faculty of Mechanical Engineering and Menagement, Poznan University of Technology, \\ Piotrowo 3, 61-138 Poznań, Poland \\ czesław.jermak@put.poznan.pl
}

received 3 November 2015, revised 27 February 2017, accepted 6 March 2017

\begin{abstract}
In the paper, the flow-through phenomena in the air gauge are under discussion form the thermodynamic and gasodynamic perspective. The main elements of the cascade are considered the inlet nozzle (restriction), measuring chamber and the measuring nozzle with the measuring slot (displacement between the nozzle head and measured surface). The purpose of the analysis was to point out the impact on the metrological characteristics of the air gauge. In particular, attention was paid to the airflow through the measuring slot. Here, the complex phenomena take place, among others the supersonic areas and a "bubble ring," which cause discontinuity and hysteresis in the static characteristic. On the other hand, the air stream expansion after the restriction (inlet nozzle) is observed in the measuring chamber. The point of the above discussion was to work out some recommendation on the nozzles geometry and the localization of the back-pressure measuring point in the chamber.
\end{abstract}

Key Words: air gauges, static metrological properties, flow-through phenomena, pressure fluctuations

\section{INTRODUCTION}

The air gauges are the excellent tools for accurate noncontact measurement (Schuetz, 2015). The merits of the air gauging are commonly known: cleaning of the measured surface during the measurement and hence indifference to the dirt and technological liquids, as well as insensitivity to the vibrations. That was the reason of wide implementation of the air gauging in the inprocess measurement, especially during the grinding and honing processes (Vacharunukul, Mekid, 2005). There are, however, some limitations in the air gauge applications, imposed by relatively short measuring range and average dynamical characteristics (Jermak, Rucki, 2016).

Metrological characteristics could be influences by modification of geometry (dimensions and shape) of the flow-through channels (Jermak, 2010). It is possible because of the gasodynamic and thermodynamic phenomena that take place when the air stream passes through the nozzles, measuring chamber, and eventually expands in the measuring slot. In fact, some of those phenomena have got a negative impact worsening metrological characteristics of the air gauge. Thus it is crucial to examine those phenomena and to undertake proper actions to reduce or eliminate their negative impact.

\section{AIR GAUGING PRINCIPLE}

The main principle of air gauging has been described scientifically as early as in 1932 (Tanner, 1958), and it is based on the influence of the geometrical value (the slot between the jet and the measured surface) on the flow-through parameters. Perhaps the most common type of the air gauge applied today is the onecascade device presented in the Fig. 1. It consists of inlet nozzle or restriction (1), measuring chamber (2) and measuring nozzle (3). Sometimes the measuring head may contain two or more measuring nozzles of equal geometry and dimensions. Multiple nozzle heads allow to perform measurement of several diameters or geometric conditions, e.g. two diameters and taper, squareness (perpendicularity) gauges, etc. (Schuetz, 2015).

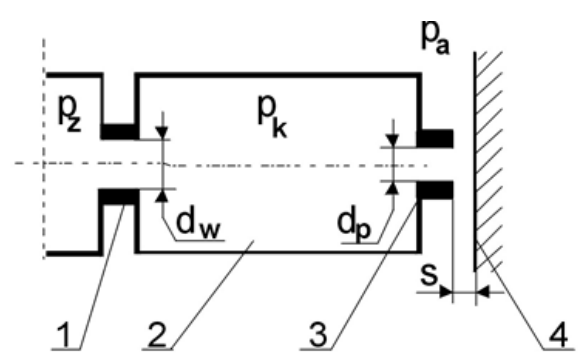

Fig. 1. Operating principle of one-cascade back-pressure open jet air gauge (Jermak and Rucki, 2012)

In open jet solutions, the outlet surface of the measuring nozzle is restricted with the surface of the measured detail (4), so the expansion of the air stream takes place in the slot $s$ between the nozzle (3) and surface (4). The feeding air is cleaned and filtered according to the requirements of the second class air quality (Zelczak, 2002). The simple constructions require high accuracy of the feeding pressure $p_{z}$ stabilization. The value of the backpressure $p_{k}$ in the measuring chamber depends on the actual slot width $s$ (Bokov, 2011)and the pre-set dimensions of the inlet nozzle $d_{w}$ and measuring nozzle $d_{p}$. 


\section{FLOW-THRUGH PHENOMENA IN THE AIR GAUGE ELEMENTS}

\subsection{Inlet nozzle}

The inlet nozzle is the integral part of the air gauge which restricts the feeding air stream setting the basic metrological characteristics of the air gauge, namely sensitivity and measuring range. In case of the unchangeable restrictions, the inlet nozzles are typically of cylindrical orifice with length $l_{d w}$ anddiameter $d_{w} \in<$ $0.5 \div 2.0>\mathrm{mm}$.

The inlet of the restriction may be shaped in different ways. In case of inexpensive nozzles, the hole may be simply drilled, a conical contraction or a simple roundings may be made (Tesar, 2010). More advanced curvature is applied in a supersonic nozzles (Tesar, 2008). Fig. 2presents the most typical solutions known and applied in air gauges for decades:

- sharp edge nozzle (perpendicular),

- conic edge nozzle,

- curved line (profiled nozzle).

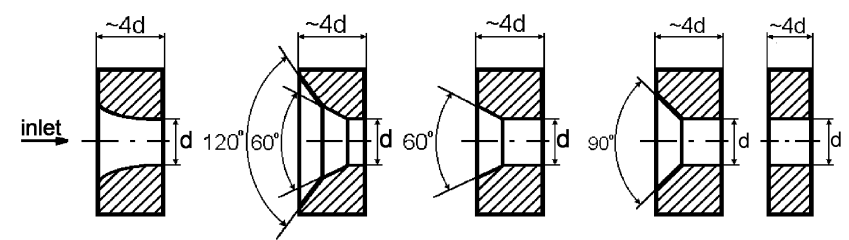

Fig. 2. Typical edges of the inlet nozzles applied in the air gauges, $L_{d w} \approx 4 \mathrm{~d}($ Dejč, 1961)

It has been found decades ago that in any type of the nozzle, the actual airflow differs from the theoretical (ideal) one. It is so because the velocity distribution in the outlet intersection is unsteady, the friction consumes some energy, and the inlet edge shape has its impact on the flow (Dejč, 1961). To calculate the actual mass flow, the flow coefficient is commonly used which is to correct the isentropic flow:

$\alpha_{w}=\frac{q_{m w}}{q_{m_{t} w}}$

where: $q_{m w}$ - mass flow, $q_{m_{t} w}$ - theoretical mass flow for the isentropic process (with no losses).

The flow coefficient $\alpha_{w}$ covers the influence of all factors which may cause the difference between the actual and theoretical mass flow, including variations of the velocity and density, stream contraction and friction in the flow channel. For any real element the flow coefficient is always $\alpha_{w}<1$.

Sharp edge nozzles (Fig. 2) generate very large variety of the flow coefficient dependent on the mass flow. It is caused by the reduced actual flow intersection as a result of the stream contraction. Moreover, when the relative length of the nozzle $L_{d w}=$ $l_{d w} / d_{w}$ is large, the friction plays more important role (Lammel and Osiadacz, 1974; Idel'čik, 1960).

When the inlet edge is conical, the variation range of the actual stream diameter is smaller, and the value aw becomes stable in the large range of the mass flow values (Breitinger, 1969). In that case, contraction is substantially smaller and the stream fills in the channel, so the flow is more sensitive to the influence of the layers close to the orifice surface, especially when the length of the nozzle $l_{d w}$ increases. The coefficient aw is dependent on the angle of the cone. If the orifice of the nozzle is very small $\left(d_{w}<\right.$ $<1 \mathrm{~mm}$ ), the layer close to surface may block the entire flow through. Then the coefficient $\alpha_{w}$ is substantially decreased.

The most advantageous function of the $\alpha_{w}$ coefficient versus pressure ratio $\beta_{w}$ could be achieved for the profiled nozzles (Fig. 3). Pressure ratio means the ratio of the absolute pressure before the restriction to the absolute pressure after it, which means for the inlet nozzle in the air gauge $\beta_{w}=p_{k} / p_{z}$. When the profile is very well and the losses are negligibly small (rapid expansion process could be approximated as adiabatic isentropic) (Dejč, 1961; Zalmanzon, 1971), then the maximal mass flow could be reached with the flow ratio close to the first critical one $\beta_{k r 1}=$ 0.528 .

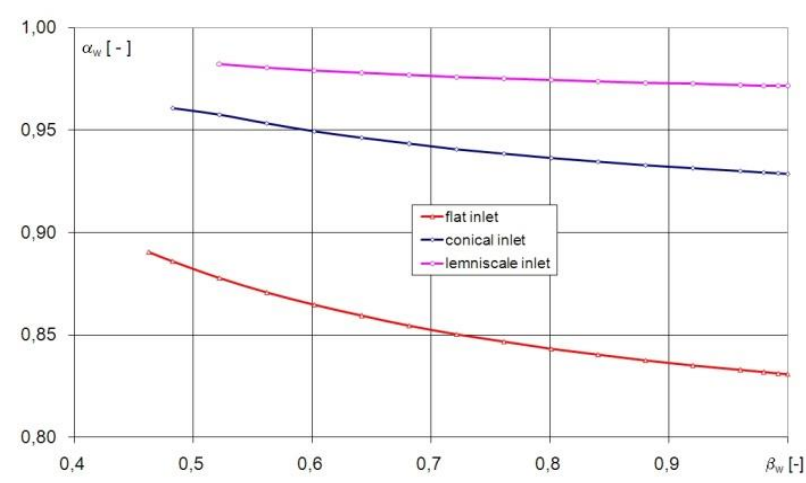

Fig. 3. Function of the aw coefficient versus pressure ratio $\beta_{w}=p_{k} / p_{z}$ for the inlet nozzles of the diameters: $d_{w}=1.002 \mathrm{~mm}$ - sharp edged; $d_{w}=0.984 \mathrm{~mm}$ - conic edge; $d_{w}=1.002 \mathrm{~mm}-$ Bernoulli's lemniscate

\subsection{Flapper-nozzle area}

In the air gauge, the flapper-nozzle area is the place where the measured dimension represented by the value $s$ comes to interactions with the air gauge nozzle to convert the dimension into the flow parameter (Zelczak, 2002), which could be e.g. backpressure $p_{k}$ or the volume $q_{v}$. The measuring nozzle could be analyzed only in interaction with the flapper surface, because those two elements determine the outflow surface and their cooperation effects with the restriction of the flow.

However, the flow through the flapper-nozzle area consists of two different stages: first the stream passes the jet with the diameter $d_{p}$ and next it expands in the measuring slot $s$. The flow is very complicated, because of the rapid change of the stream direction and the way of expansion. In front of the jet, the stagnation point appears, and around it the boundary layer is formed. This layer thickness depends on many factors, mainly on the jet diameter $d_{p}$ and Reynold's number. For the relative displacement $\mathrm{s} / d_{p} \leq 4$ it is constant (Oleśkowicz-Popiel, 1981).

The boundary layer appears also close to the measuring nozzle front surface, which decreases the actual outlet surface. In this small area, large differences of pressure may appear causing supersonic local flows and even supersonic zones (Kazimierski and Krysiński, 1981). As a result, we deal with the thermodynamic flow process of the compressible medium, where along with sufficient velocity variety takes place highly diversified temperature distribution, where the air density is very difficult to be determined. The rapid change of the flow direction near the stagnation point provides highly complex mechanism of the energy transport, 
because the kinetic energy of the stream falling on the flapper should be transformed into the rest enthalpy in the stagnation point, which in turn causes the increase of the local static pressure. On the other hand, the expansion of the air in the slot causes the radial flow and very large losses of the pressure. All the phenomena are very difficult to be described and visualized experimentally, and the measurement of the particular local vales is difficult because of the very small dimensions of the flow area. Both quality and quantity description of the flow in those conditions are very complicated.

In terms of pressure losses, there could be pointed out several zones in the flapper-nozzle area, where those losses are most evident (Lotze, 1965; Jermak and Rucki, 2012). These are shown in the Fig. 4 and included into the formula (3) below.

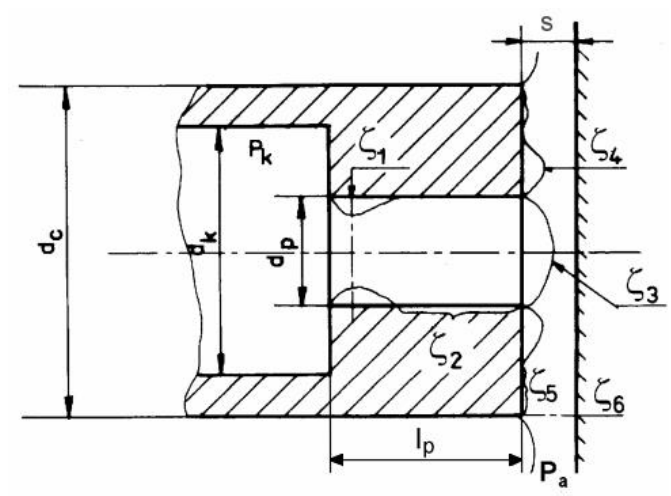

Fig. 4. Pressure losses in the flapper-nozzle area (Lotze, 1965)

The loss coefficient $\zeta$ has been defined by (Prosnak, 1971) as a ratio of the pressure difference caused by losses $\Delta p_{s t r}$ divided by mean dynamic pressure in the analyzed point or intersection:

$\zeta=\frac{2 \Delta p_{s t r}}{\rho C^{2}}$.

The negative impact of the loss of the pressure $\Delta p_{\text {str }}$ on the sensitivity of the air gauge should be minimized. Different components of the coefficient $\zeta$ may affect the back-pressure $p_{k}$ in different way and with different strength. So to express the overall pressure loss related to the mean pressure in the measuring slot $s$ the following formula has been proposed (Lotze, 1965):

$\zeta_{c}=16 \frac{d_{c}^{2} s^{2}}{d_{p}^{4}}\left(\zeta_{1}+\zeta_{2}+\zeta_{3}\right)+\left(\left[\frac{d_{c}}{d_{p}}\right]^{2} \zeta_{4}+\zeta_{5}+\zeta_{6}\right)$.

The first component of the formula (3) contains losses in the nozzle itself (see $\zeta_{1}, \zeta_{2}$ and $\zeta_{3}$ in the Fig. 4), while the second is related to the slot between the measuring nozzle head and the flapper surface. The coefficient $\zeta_{1}$ represents the stream contraction effect after the rapid decrease of the stream diameter. (Lotze, 1965) has proposed the formula to calculate its value:

$\zeta_{1}=\left(\frac{1}{\mu_{k}}-1\right)^{2}$

where: $\mu_{k}-$ coefficient of contraction.

The variation range of the contraction coefficient $\mu_{k}$ depends on the profile of the inlet part of the nozzle. It lays in the range of $\langle 0.61 \div 0.99\rangle$, imposing the variety range of the loss coefficient: $\zeta_{1} \in<0.0001 \div 0.41>$. Thus, it is possible to reduce $\zeta_{1}$ applying profiled inlet area with large roundness.

Further pressure losses are the effect of the friction along the flow channel. The coefficient $\zeta_{2}$ depends on the Reynolds number
Re and on the length of the nozzle $l_{p}$. It is hardly expected the laminar flow in the working range of the slot widths $s$, so the following formula could be applied (Lotze, 1965):

$\zeta_{2}=\frac{0,316}{\sqrt[4]{R e}} \frac{l_{p}}{2 s}$.

The local losses described by the coefficient $\zeta_{3}$ correspond with the expansion of the air leaving the jet. According to (Lotze, 1965), overall impact of those three components $\left(\zeta_{1}, \zeta_{2}\right.$ and $\left.\zeta_{3}\right)$ is rather small and do not exceed $8 \%$.

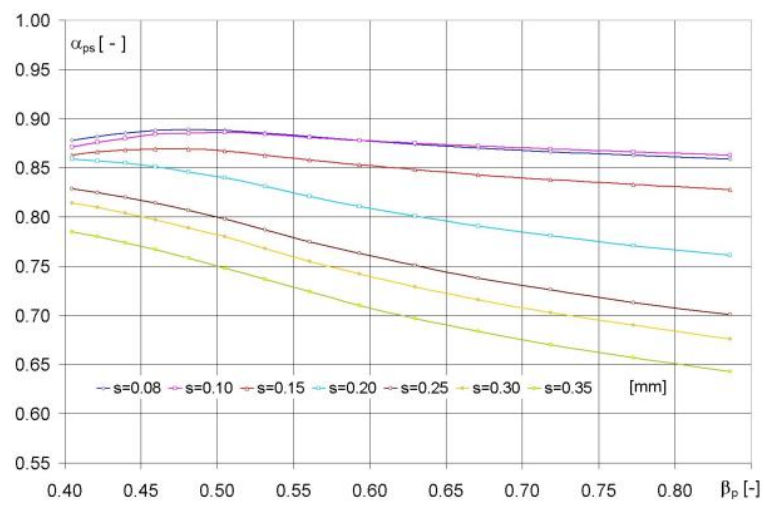

Fig.5. Graph of the flapper-nozzle flow coefficient $\alpha_{p s}$ versus flow ratio $\beta_{p}$ for different slots $s$, the air gauge $d_{p}=2.008 \mathrm{~mm}, D_{c}=1.5$

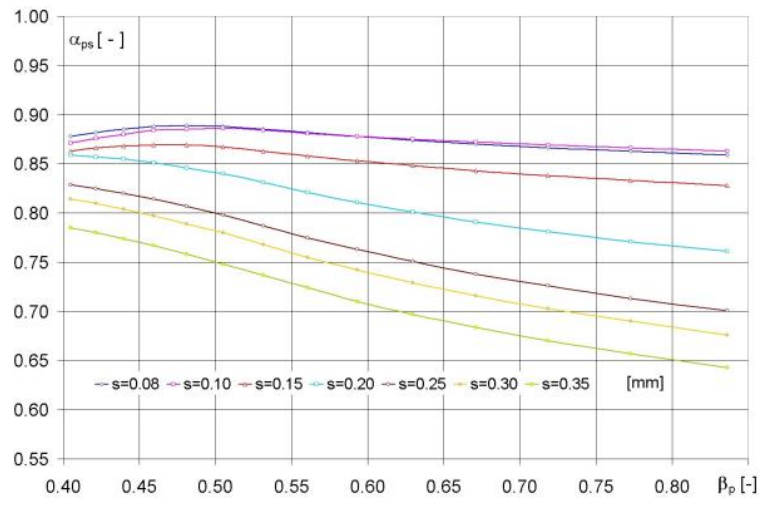

Fig. 6. Graph of the flapper-nozzle flow coefficient $\alpha_{p s}$ versus flow ratio $\beta_{p}$ for different slots $s$, the air gauge $d_{p}=2.008 \mathrm{~mm}, D_{c}=3.0$

Like in case of the stream contraction and coefficient $\zeta_{1}$, rapid change of the flow direction after the nozzle edge causes the appearance of a "bubble ring" which results with the narrower than $\mathrm{s}$ actual flow. Coefficient $\zeta_{4}$ is to represent that phenomenon, and when those "bubble rings" appear further at larger diameters as a result of the strike waves, additional pressure losses are described as $\zeta_{5}$, covering friction losses as well.

Finally, when the stream of expanding air reaches the outer edge of the nozzle $d_{c}$, it is no more restricted by the slot. Here takes place the last pressure loss $\zeta_{6}$ down to the atmospheric pressure pa, but its value is relatively small compared to $\zeta_{4}$ and $\zeta_{5}$.

To determine exact losses in the slot area $\left(\zeta_{4}, \zeta_{5}\right.$ and $\left.\zeta_{6}\right)$, the flow should be precisely characterized in all the crucial zones. It is a difficult task, thus the flow coefficient for the flapper-nozzle area aps appears to gain very different values. It should be noted that it varies not only for different slot widths $s$ (and, hence, relative 
pressure $\left.\beta_{p}=p_{a} / p_{k}\right)$, but also for different outer diameters of nozzle, expressed by relative value $D_{c}=d_{c} / d_{p}$. Figs. 5 and 6 show the corresponding graphs.

a)

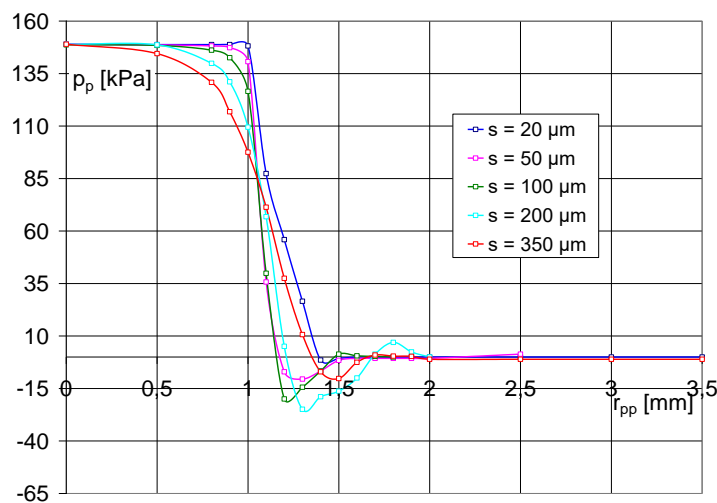

b)

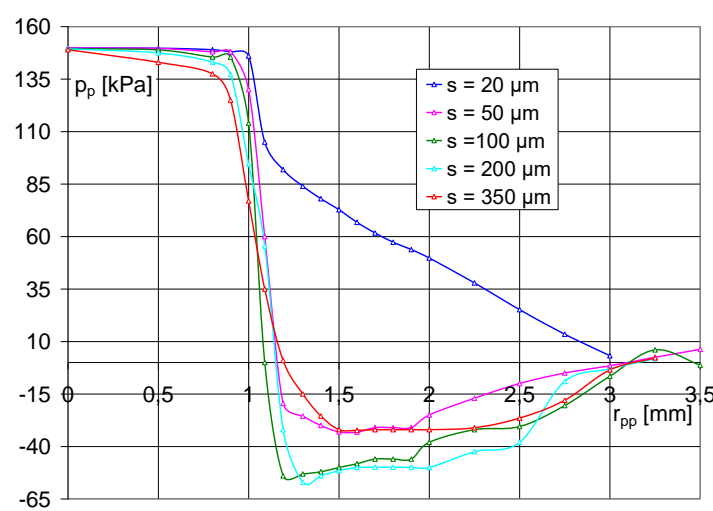

c)

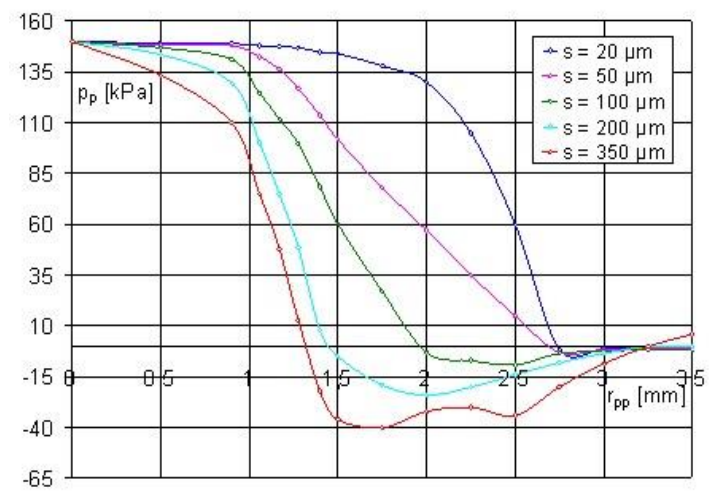

Fig. 7. Pressure distribution on the flapper surface for different measuring nozzles: a) flat narrow, $D_{c}=1.25$; b) flat wide, $D_{c}=3$; c) profiled, $d_{p}=2.008 \mathrm{~mm}, d_{w}=0.802 \mathrm{~mm}$

The experimentally obtained values of the local pressure on the flapper surface provide some additional information on the phenomena in the measuring slot. Following air gauges underwent examinations:

- inlet nozzle $d_{w}: 0.802 \mathrm{~mm}$,

- measuring nozzle $d_{p}=2.005 \mathrm{~mm}$,

- feeding pressure $p_{z}=150 \mathrm{kPa}$,

- the measuring nozzle head surfaces: flat narrow $\left(D_{c}=1.25\right)$, flat wide $\left(D_{c}=3\right)$ and profiled according the lemniscate.

The results of the experiments led to the following conclusions:
- the local pressure $\mathrm{p}_{\mathrm{p}}$ on the flapper surface measured on the radius $r_{p p}$ could be described as:

$p_{p}\left(r_{p p}\right)=f\left(p_{z}, s, d_{p}, d_{p}, D_{c}\right)=$ const,

- relatively large variations of the pressure appear in the flow area between outer and inner diameters of the nozzle head $\left(d_{c}-d_{p}\right)$; it is there where "bubble rings" could be found, which are generated by sharp edge of the nozzle, rapid change of the flow direction and high velocity of the stream;

- large pressure gradients take place close to the inner edge of the nozzle, especially when the slot s is small;

- nozzles of narrow head surface generate rapid pressure change in the area of the orifice and relatively small subatmospheric pressure on the flapper surface (Fig. 7a). Large pressure gradient causes the expansion, which leads to maximal velocity with minimal pressure. It is highly probable that in this very area the air velocity reaches supersonic values. Further the velocity quickly decreases and the pressure on the flapper surface comes close to the atmospheric one.

- in case of the wide head nozzles no sub-atmospheric pressure was noted on the flapper surface for the slots smaller than $\mathrm{s}=20 \mu \mathrm{m}$ (Fig. $7 \mathrm{~b}$ ), and the pressure gradient along the head was small.

- the outlet edge of the profiled nozzle is placed higher than the outer edge. That is why the stagnation zone is much larger. As a result, the stream changes its direction smoothly, and the sub-atmospheric pressure on the flapper surface appears only for slots larger than $150 \mu \mathrm{m}$ (Fig. 7c). For the larger slots, the phenomenon of the local stream slowdown effected with the appearance of the zones with higher pressure.

- in the area larger than restricted with the nozzle head, some pressure could be noted on the flapper surface, which may prove the dynamic effect of the stream slowdown.

- in all examined cases, the radius $r_{p p}$ of the maximal subatmospheric pressure was close to $(0.60 \div 0.70) d_{p}$.

\subsection{Pressure distribution in the measuring chamber}

The air gauge's measuring chamber is the area between the inlet and measuring nozzles. Its task, on the one hand, is to dissipate the energy of the air stream leaving the inlet nozzle, and on the other hand, to provide the conditions for proper back-pressure measurement. It is required to ensure the minimal fluctuation of the pressure in the measuring point. If the chamber did not provide full expansion of the air stream, it would cause dynamical effects in its end part close to the measuring nozzle. When this requirement is met, the overall pressure in the area is equalized and could be measured in the point placed on the side surface. Theoretical (hypothetic) structure of the flow through the measuring chamber is shown in the Fig. 8.

The most complicated is the description of the flow in the measuring chamber area close to the inlet nozzle outlet surface. The expanding stream generates here some whirls and backflows caused by the small pressure with gradient both along the stream line, and perpendicular to it.

The velocity field in the perpendicular cross-section of the stream consists of the constant velocity segments and the transitional zone (turbulent boundary layer). The flow characteristic, in particular, is the variation of the velocity along the axis of the 
circulation area. The boundary layer gradually widens from the outlet orifice surface, and at the certain distance $l_{s}$ reaches its maximal width. The pressure distribution changes accordingly, as it is shown in the Fig. 8. When the stream leaves the nozzle, its pressure is $p_{1^{\prime}}$, and in the minimal intersection $\left(p_{\text {min }}\right)$, there is the minimal pressure $p_{1^{\prime} \text { min }}$.
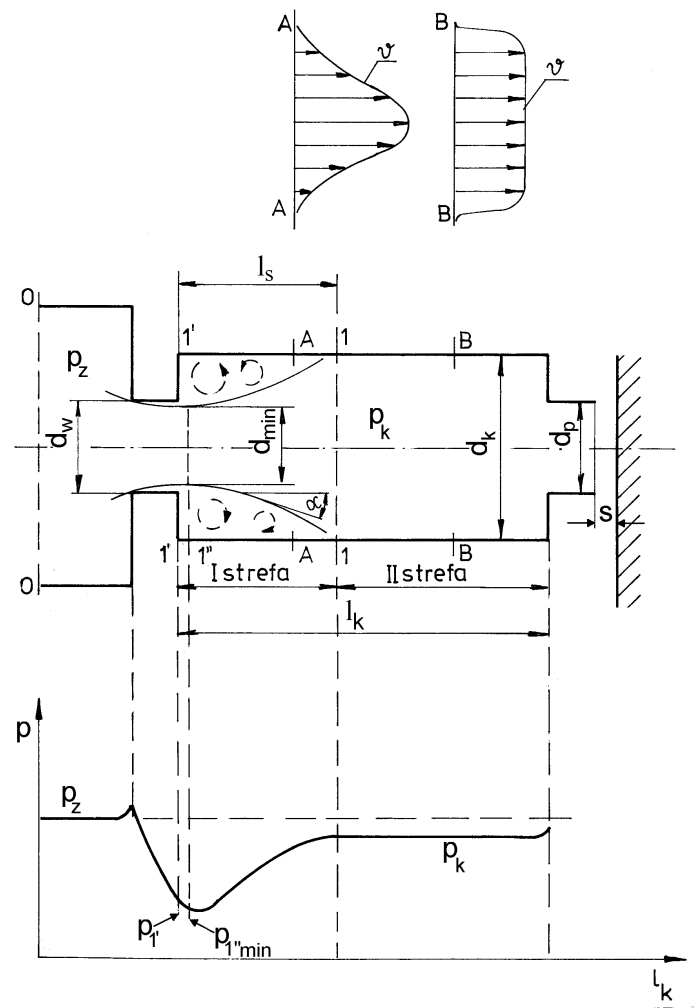

Fig. 8. Hypothetical structure of the stream expansion in the measuring chamber of length $l_{k}$ and diameter $d_{k}$

Contraction of the stream $d_{\min } / d_{w}$ is dependent on the geometry of the inlet nozzle and its dimensions, and in case of the profiled jets it could be close to 1. After the minimal cross-section marked in the Fig 8 as $11^{\prime \prime}-1$ ", the increase of the pressure could be observed up to the maximal pressure in the cross-section 1-1. Further from the minimal cross-section, the pressure varies not much revealing weak trend to decrease, which can be explained with the friction inside the chamber. The distribution of the velocity and pressure in the chamber depend on the feeding pressure $p_{z}$, thus on the mass flow $q_{m}$, too. Of course, dimensions of the measuring chamber are of significant importance as well.

The most important form the designer's perspective is to know the dimensionless values that determine the geometry of the chamber: $d_{k} / d_{w}$ and $l_{k} / d_{k}$. The minimal length of the measuring chamber is restricted by the relation $l_{s}<l_{k}$ for the assumed ratio $d_{k} / d_{w}$. This way the minimal dimensions of the measuring chamber could be estimated. Then it could be assumed that the dynamical effects near the measuring nozzle are negligibly small, and the mean velocity here is no larger than $15 \%$ of the velocity close to the inlet nozzle. Under such conditions, the authors Dejč (1961) and Zalmanzon (1961) recommend the following dimensions:

$l_{k} \approx(4 \div 5) d_{k} ; d_{k} \approx 7 d_{w}$
For the experimental research it was assumed to investigate the chambers of the diameter $d_{k}=10 \mathrm{~mm}$ and length $l_{k}=20,40$ and $80 \mathrm{~mm}$. The measured values were: overall pressure $\mathrm{pc}$ measured along the axis of the chamber, and the loss of the pressure $\Delta p_{k}$ on the chamber sides. The reference value was the back-pressure measured on the chamber side $0.5 \mathrm{~mm}$ far from the inlet nozzle edge. The experiments were repeated for the mass flow rates $q_{m}$ of $6 \times 10^{-4} \mathrm{~kg} / \mathrm{s}$ and $14 \times 10^{-4} \mathrm{~kg} / \mathrm{s}$, which correspond with the air gauge work at the slot width $s=50 \mu \mathrm{m}$ and 350 $\mu \mathrm{m}$, respectively. The results of experimental measurements are shown in the Fig. 9a and b.

a)

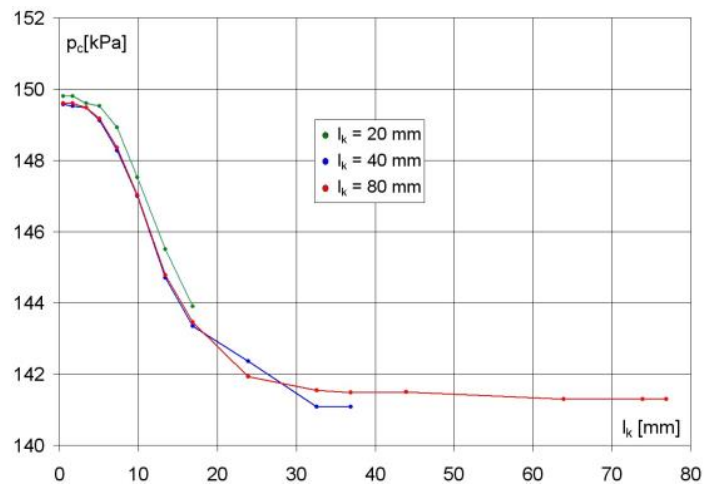

b)

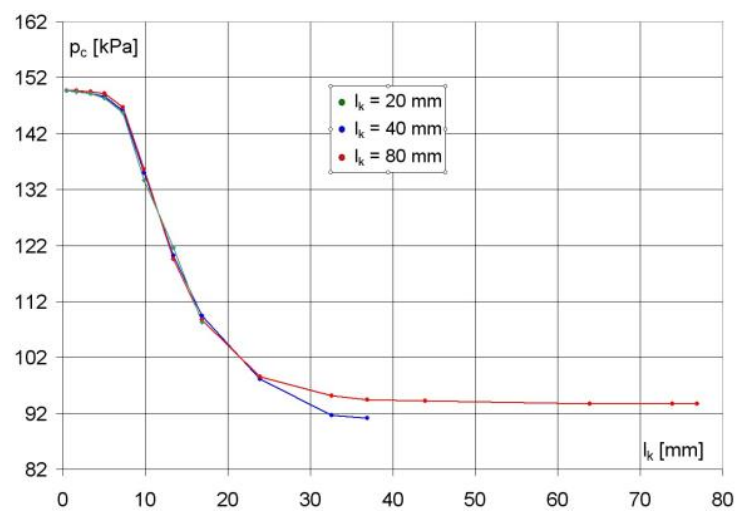

Fig. 9. Measurement results for the overall pressure pc versus chamber length $l_{k}$ for different mass flow rates: a) $q_{m}=6 \times 10^{-4} \mathrm{~kg} / \mathrm{s}$, b) $q_{m}=14 \times 10^{-4} \mathrm{~kg} / \mathrm{s}$

Analysis of the above graphs leads to the conclusion that the overall pressure is dependent on the mass flow rate and on the distance from the inlet nozzle edge. Regardless what is the mass flow rate, in the chambers of the length $l_{k}=20 \mathrm{~mm}$ the overall pressure got never stabilized. That means high flow velocity and incomplete expansion of the stream across the chamber volume. Completely different result is seen for the chamber of length $l_{k}=$ $40 \mathrm{~mm}$ and $80 \mathrm{~mm}$. In both cases, the overall pressure reached its stabilized value dependent on the mass flow at the length ca. $0.8 l_{k}$. The pressure decrease in both cases corresponded with ca. $0.45 \mathrm{kPa}$ per $1 \mathrm{~mm}$ of the chamber length for the mass flow $q_{m}=6 \times 10^{-4} \mathrm{~kg} / \mathrm{s}$ and ca. $4.0 \mathrm{kPa}$ per $1 \mathrm{~mm}$ of the chamber length for the mass flow $\mathrm{q}_{\mathrm{m}}=14 \times 10^{-4} \mathrm{~kg} / \mathrm{s}$.

The changes of the pressure $\Delta p_{s t}$ on the chamber sides versus distance from the inlet nozzle varied from $+25 \mathrm{~Pa}\left(l_{k}=40\right.$ and $80 \mathrm{~mm})$ down to $-480 \mathrm{~Pa}\left(l_{k}=80 \mathrm{~mm}\right)$ for the mass flow $q_{m}$ $=6 \times 10^{-4} \mathrm{~kg} / \mathrm{s}$ and from $+250 \mathrm{~Pa}\left(l_{k}=20 \mathrm{~mm}\right)$ down to $-3000 \mathrm{~Pa}$ $\left(l_{k}=80 \mathrm{~mm}\right.$ ) for $q_{m}=14 \times 10^{-4} \mathrm{~kg} / \mathrm{s}$ (see Fig. 10a, b) . 
a)

b)
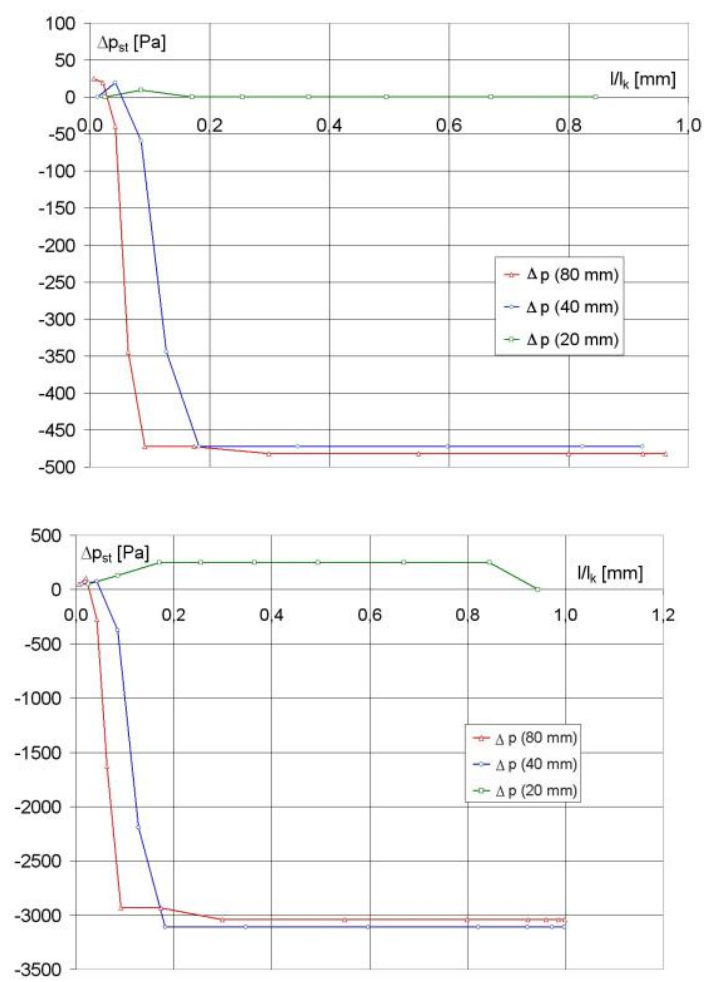

Fig. 10. The results of measurement of the back-pressure $\Delta p_{s t}$ versus the relative length from the inlet nozzle edge $l_{i} / l_{k}$ for different mass flow rates: a) $q_{m}=6 \times 10-4 \mathrm{~kg} / \mathrm{s}$, b) $q_{m}=14 \times 10^{-4} \mathrm{~kg} / \mathrm{s}$

It could be stated that in the chambers of the length $l_{k}=20$ $\mathrm{mm}$ took place the dynamic phenomenon with various intensity, but always resulting with back-pressure value $\Delta p_{s t}=0$ (for $q_{m}=$ $6 \times 10-4 \mathrm{~kg} / \mathrm{s}$ ) or $\Delta p_{s t}>0$ (for $q_{m}=14 \times 10-4 \mathrm{~kg} / \mathrm{s}$ ). Initial recommendation could be made that it is reasonable to place there the measuring point for the back-pressure $p_{k}$.

In the longer chambers, the rapid fall of the back-pressure takes place in the area from 0.1 to $0.2 l_{k}$. It is assumed that this particular point should not be chosen for the back-pressure measurement.

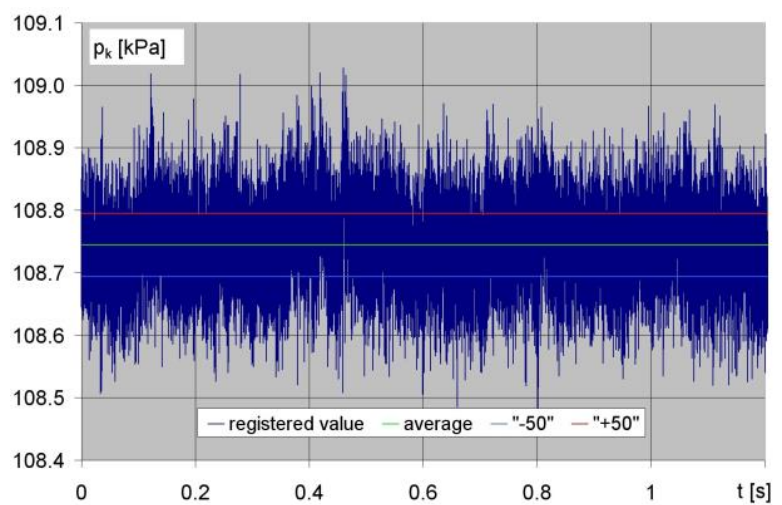

Fig. 11. Registered values of the back-pressure $p_{k}$ in the point $\mathrm{A}$; $d_{p}=2.005 \mathrm{~mm}, d_{k w}=1.210 \mathrm{~mm}, D_{c}=3, s=170 \mu \mathrm{m}$; red and blue lins correspond with the acceptable fluctuations of $\pm 50 \mathrm{~Pa}$

During the outflow process from the inlet nozzle to the chamber, strong turbulences take place. Thus, the back-pressure $p_{k}$ measured both in static and dynamic conditions never achieves stable constant value, but is a subject of strong fluctuations shown in the Fig. 11. This way the reliability of the dimensional measurement result is under question both in the adjustment process and in the inspection, because the pressure fluctuations reveal themselves in changes of the dimension indication (Rucki, 2009).

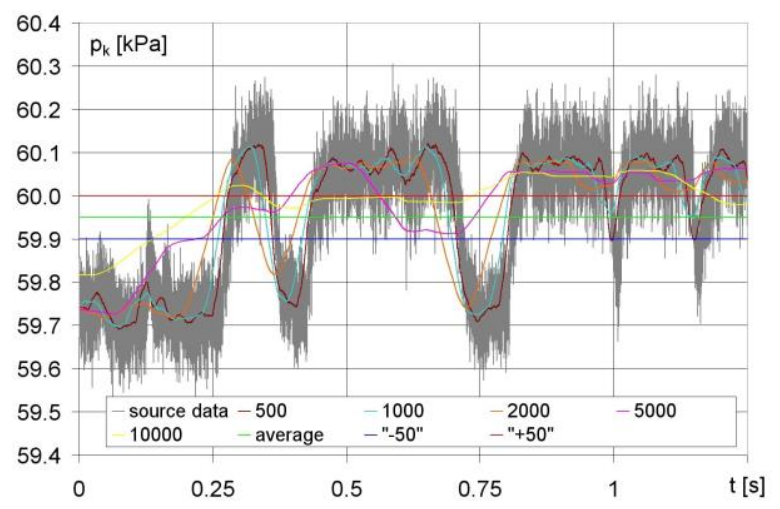

Fig.12. Fluctuations of the back-pressure $p_{k}$ in the "jump" area; the air gauge parameters: $d_{p}=2.005 \mathrm{~mm}, d_{w}=1.210 \mathrm{~mm}, D_{c}=3$

a)

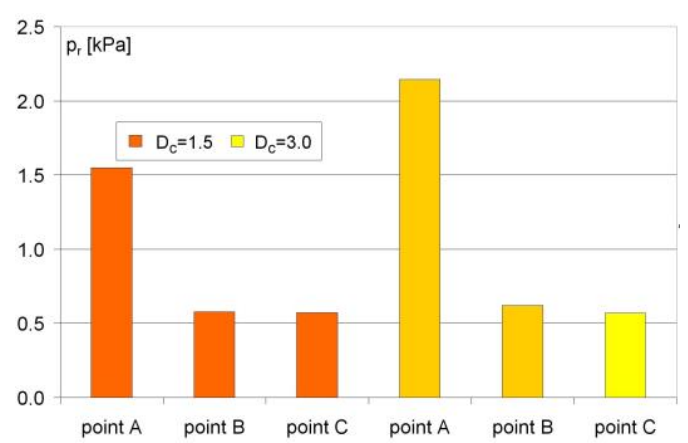

b)

c)
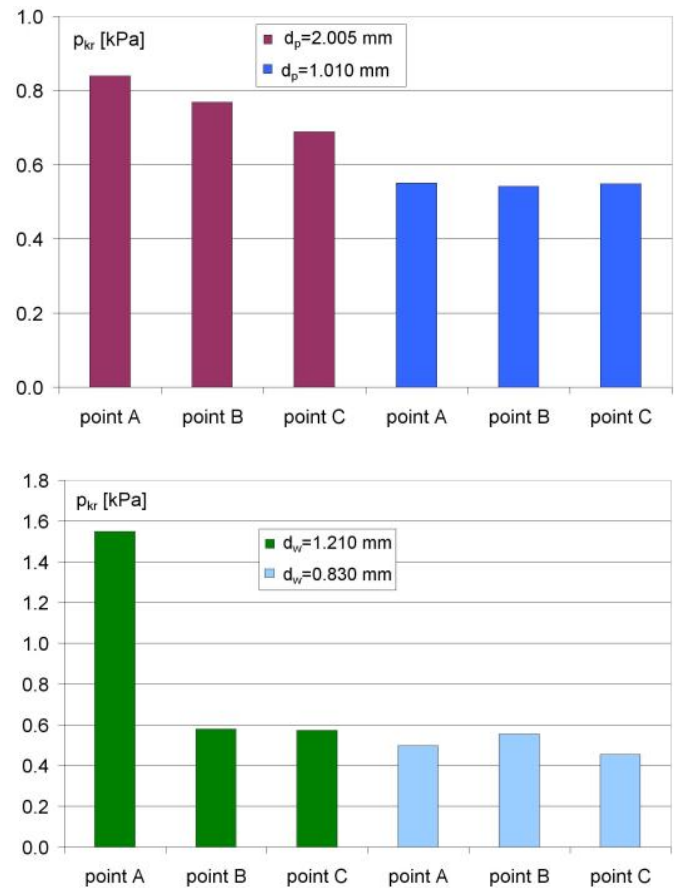

Fig. 13. Maximal range $\left(p_{r}=p_{\text {kmax }}-p_{\text {kmin }}\right)$ of the back-pressure fluctuation dependent on: a) head surface $D_{c}\left(d_{p}=2.005 \mathrm{~mm}\right.$, $\left.d_{w}=1.210 \mathrm{~mm}\right)$, b) diameter $d_{p}\left(d_{w}=1.210 \mathrm{~mm}, D_{c}=1.5\right)$, c) diameter $d_{w}$ (for $d_{p}=2.005 \mathrm{~mm}, D_{c}=1.5$ ) 
The dimensions of the nozzles used in the investigations, the measuring one (diameter $d_{p}=2.008 \mathrm{~mm}$ and head surface $D_{c}=1.5$ and 3.0$)$ and the inlet one $\left(d_{w}=0.830 \mathrm{~mm}\right.$ and 1.210 $\mathrm{mm}$ ) provided the variations of the metrological characteristics in the range that corresponded with the working conditions of the industrial air gauges.

a)

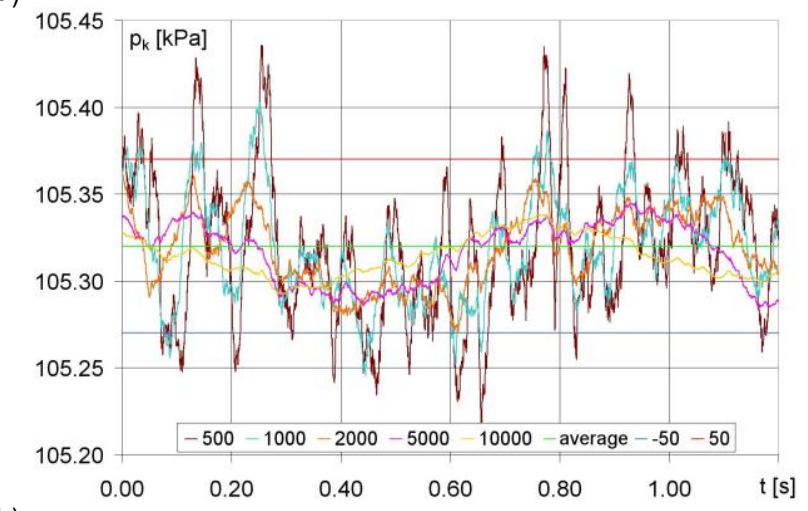

b)

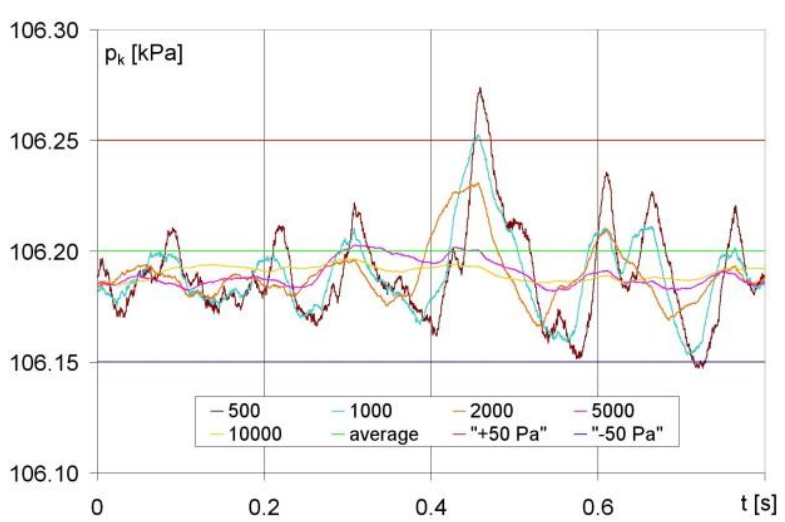

c)

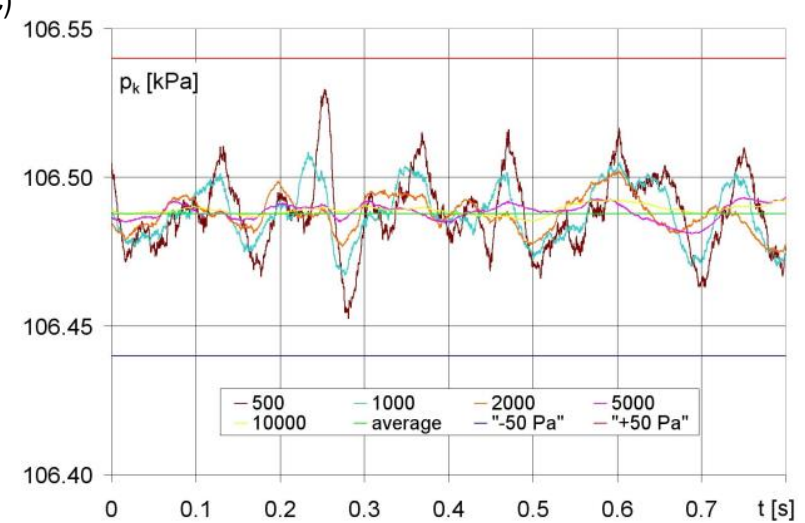

Fig. 14. The averaged signals of the back-pressure $p_{k}$ in the points: a) $A$, b) B, c) $C$ for the air gauge of $d_{p}=2.005 \mathrm{~mm}, d_{w}=1.210 \mathrm{~mm}$

The dimensions of the examined chambers were following: diameter $d_{k}=10 \mathrm{~mm}$ and length $l_{k}=70 \mathrm{~mm}$. The probing points $\mathrm{A}, \mathrm{B}$ and $\mathrm{C}$ were placed at the distance from the inlet nozzle edge $l_{k A}=10 \mathrm{~mm}, l_{k B}=40 \mathrm{~mm}$ and $l_{k C}=68 \mathrm{~mm}$, respectively. During the measurement, it could be noted that the back-pressure varied with the substantial amplitude, and two periods could be distinguished - longer ones of amplitude ca. $0.5 \mathrm{kPa}$ and high frequency ones of smaller amplitude ca. $0.3 \mathrm{kPa}$ (Fig. 12).

When those two waves are superposed, the resulting range of fluctuation of the back-pressure $p_{k}$ reaches $R=0.7 \div 0.8 \mathrm{kPa}$.
Along the walls of the chamber this process is occurring with various intensity, and reaches its peak close to the inlet nozzle (point A). To evaluate the range of the fluctuations $p_{r}=p_{\text {kmax }}{ }^{-}$ $p_{\text {kmin }}$, the investigations were repeated for 64,000 samples with sampling interval $t_{p}=0.0004 \mathrm{~s}$. It was noted that the fluctuations are dependent on the geometrical parameters of the nozzles: the head surface $D_{c}$, as well as the diameters $d_{p}$ and $d_{w}$.

The analysis of the registered fluctuations, like shown in the Fig. 11, confirmed the earlier statement that there are significant turbulences in the area close to the inlet nozzle. It is particularly obvious in case of the air gauges with large diameters of the nozzles. The example shown in the Fig. 13 suggests also that the increase of the head surface $D_{c}$ from 1.5 up to 3.0 could cause large fluctuation of the back-pressure in the point $A$. In other probing points of $p_{k r}$, the fluctuation level got stabilized and reached the value dependent on the measuring and inlet nozzles diameters. Certainly the best reduction of the fluctuations was achieved for the air gauge with the measuring nozzle $d_{p}=2 \mathrm{~mm}$ and inlet nozzles smaller than $1 \mathrm{~mm}$. The localization of the measuring point was then of secondary importance.

However, the recommended localization of the measuring point is not enough to achieve the signal of possibly minimal fluctuation. The signal has to be averaged in order to make the amplitude fit the required range. Considering the high requirements on the uncertainty of the slot width determination, the maximal acceptable deviation of the back-pressure signal was assumed to lay in the range $\pm 50 \mathrm{~Pa}$. In the graphs (Fig. 14), those upper and lower limits are represented with red and blue lines, respectively. The green line corresponds with the mean value.

Fig. 14 presents the graphs for the registered back-pressure in the measuring points $A, B$ and $C$, obtained for different numbers of samples (from 500 up to 10,000) intended to be averaged. For the sampling time of $t_{p}=0.0004 \mathrm{~s}$ the time span $t_{u}$ for the averaging process is from $0.2 \mathrm{~s}$ to $4 \mathrm{~s}$.

Again, the dependence of the point localization and the fluctuation level is clearly seen. Certainly the largest range of fluctuations take place close to the inlet nozzle. When the acceptable fluctuation must not exceed $\pm 50 \mathrm{~Pa}$, in the point $A$ the averaging time must be $t_{u}=0.8 \mathrm{~s}$, while in the point $C$ some 500 samples is enough $\left(t_{u}=0.2 \mathrm{~s}\right)$.

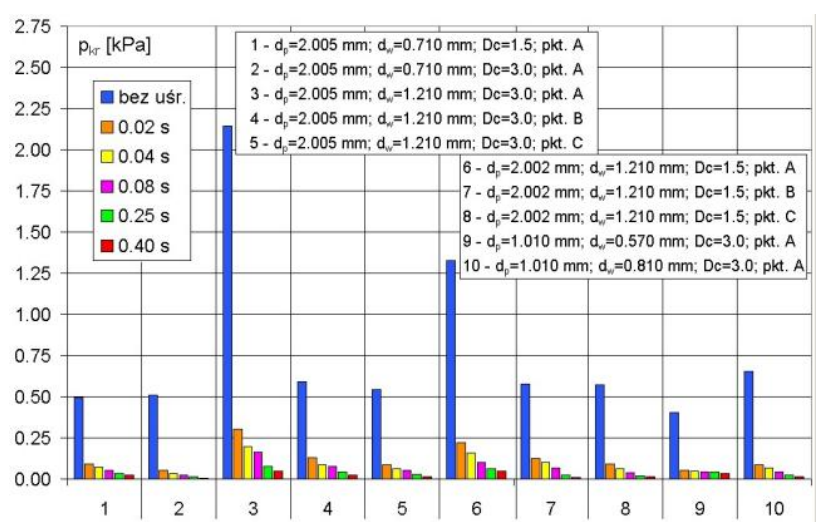

Fig. 15. Averaging time and the fluctuation amplitude $p_{r}$ for different configurations of the air gauges and probing points localization (numbers 1-10 correspond with particular configurations)

The dimensions of the nozzles in air gauges also have their impact on the fluctuations. The earlier conclusion against the 
nozzles of large diameters found its confirmation also in case of the configuration \#3 shown in the Fig. 15. That comes to be true both in case of the rough measuring signals and the averaged ones. In the extremes, the range of the fluctuations reached values over $2 \mathrm{kPa}$, which corresponded with the slot width ca. $5 \mu \mathrm{m}$, or $5 \%$ of the measuring range. The decrease of the head surface down to $D_{c}=1.5$ and the change of the probing point localization allowed to decrease fluctuations down to $25 \%$ of maximal value. However, the averaging process appears to be necessary anyway.

\section{DISCUSSION ON THE DISCONTINUITY OF THE AIR GAUGES CHARACTERISTICS}

The main metrological properties of the air gauge are described by its static characteristics which describes the function of the back-pressure $p_{k}$ versus slot widths. This function is influenced by many geometrical parameters, as well as thermodynamic and flow-through conditions. From the metrological perspective, the changes in the multiplication (sensitivity) can provide much important information on the air gauge. In the article, the module of sensitivity $|K|=f(s)$ is considered. ing:

Typically, the sensitivity of an air gauge is defined as follow-

$$
K=\lim _{\Delta s \rightarrow 0}\left(\Delta p_{k} / \Delta s\right) \approx \frac{d p_{k}}{d s}
$$

where: $\Delta \mathrm{p}_{\mathrm{k}} \quad$ - increase of the back-pressure caused by the increase of the slot width, $\Delta s \quad-\quad$ increase of the slot width in the linear area of the function.

In many cases, it was found that the actual static characteristics reveal discontinuity of the back-pressure $\mathrm{p}_{\mathrm{k}}$, which affects the metrological properties of the air gauge shortening its measuring range. Fig. 16 provides the example of the phenomenon, where around the slot width $s=280 \mu \mathrm{m}$ one can see the huge "jump" of the sensitivity and the hysteresis in the $\mathrm{p}_{\mathrm{k}}=f(s)$ function. Perhaps the first scholar who paid attention to the phenomenon was (Markow, 1971).

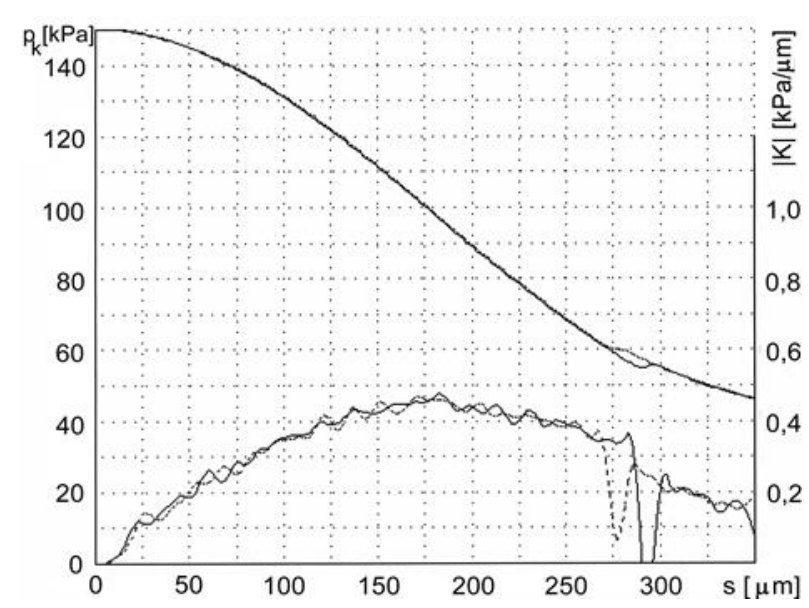

Fig. 16. Static characteristic $s p_{k}=f(s)$ and sensitivity graph $|K|=$ $f(s)$ of the air gauge with $d_{w}=2.002 \mathrm{~mm}$, $d_{p}=1.210 \mathrm{~mm}$, and $D_{c}=3$

The discontinuity provides some problem which may be solved in two different terms. The first term is the understanding of the phenomena that cause the problem and identification of the main sources. The second term is the practical elimination of the discontinuity or removal it from the linear area of the function. To do it, one must choose proper dimensions and shape of the air gauge elements.

The authors (Crnojevic et al., 1997) suggested that the discontinuity was caused by the diagonal strike wave that appeared in the outlet area of the measuring nozzle close to the flapper surface. As a result, the pressure losses increased and hence the airflow decreased. Similar explanations were proposed by other researchers (Breitinger, 1969; Zelczak, 2002).

It can be stated that the discontinuity is caused by many factors like feeding pressure $p_{z}$, relation between nozzle dimensions $d_{w} / d_{p}$, measuring slot width $\mathrm{s}$ and the outer diameter of the measuring nozzle $d_{c}$. It seems reasonable to look at the phenomenon from such a practical perspective.

Fig. 16 above presents the results of the investigations on the air gauge of following dimensions: $d_{p}=2.002 \mathrm{~mm}, d_{w}=1.210$ $\mathrm{mm}$ and $D_{c}=3$ (Project Report, 2001). In the sensitivity graph $|K|=f(s)$ a series of areas can be pointed, where different kinds of the airflow take place. The laminar flow turns to the turbulent one in the inlet nozzle when the slot width is $s=20 \div 25 \mu \mathrm{m}$, which results with some "jump" in the sensitivity graph. Further fluctuations of the sensitivity, especially the ones around the slot widths sca. $55 \mu \mathrm{m}$ and $70 \mu \mathrm{m}$, correspond with the similar changes of the flow in the measuring nozzle orifice and in the slot, respectively (Breitinger, 1969). When laminar flow turns into the turbulent one, some decrease of the sensitivity could be seen. In the range of the slots $s=275 \div 300 \mu \mathrm{m}$ take place contractions of the air stream that lead to the disturbance of the static characteristics. Rapid increase and then decrease of the back-pressure form a hysteresis. High dynamics of the phenomenon attracts attention (Fig. 17): the "jump" lasts ca. $0.005 \mathrm{~s}$, which corresponds with the ratio of $600 \mathrm{kPa} / \mathrm{s}$.

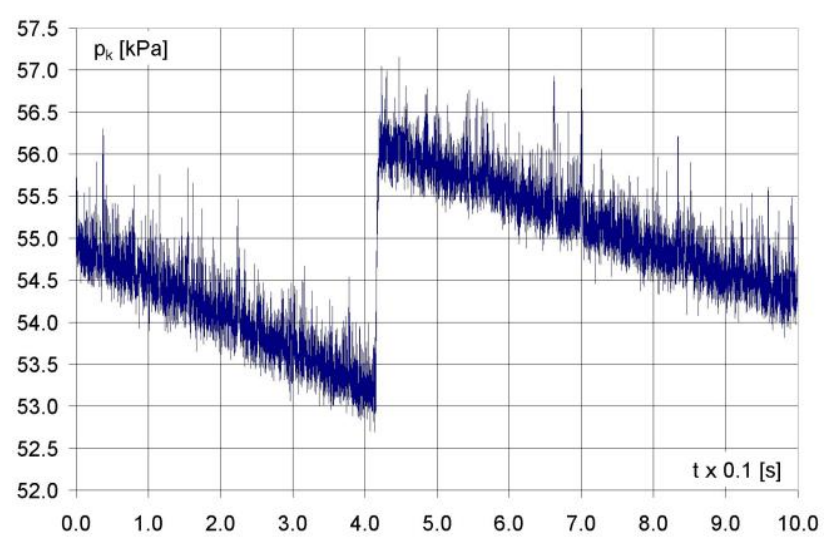

Fig. 17. Fragment of the static characteristics from the Fig. 16 close to $s=290 \mu \mathrm{m}$ obtained when the slot was increased

The graphs presented in the Fig. 16 and 17 were obtained through slow controlled movement of the flapper surface in front of the measuring nozzle (see Jermak and Rucki, 2012). The direction of the "jump" between $275 \mu \mathrm{m}$ and $300 \mu \mathrm{m}$ depends on the condition, is the flapper surface moving towards or away from the nozzle. Since the discontinuity appearance depends on the slot width s, static characteristics become ambiguous which leads to the decrease of the measuring range. 


\section{CONCLUSIONS}

Metrological characteristics of the air gauges are formed by the range of the thermodynamic and gasodynamic phenomena that take place in the flow-through elements like nozzles, measuring chamber or flapper-nozzle area. In every element some losses of energy and pressure occur, which may be expressed with the flow coefficient. The coefficient is dependent among others on the dimensions and shape of the nozzle. In case of the inlet nozzles, it is recommended to form the inlet surface with curved profile, which could cause some change in the flow coefficient.

The source of the main disturbances of the static characteristics, however, lays in the phenomena located in the flapper-nozzle area. The airflow here is very complicated because of the flow direction change around a stagnation point. Large differences of the local pressures may lead to the supersonic local flow. On the flapper surface, the areas with pressure substantially below the atmospheric pressure appear, temperature falls and the phenomenon of the transition of the flow from the surface of nozzle to the flapper surface and back again.

In the measuring chamber, the flow is mainly characterized by the pressure distribution. The pressure on the chamber walls reaches its minimal value in the maximal cross-section of the air stream. In the area close to the inlet nozzle edge, recirculation area appears where strong turbulences take place. Hence, the back-pressure measuring point is recommended to be placed at some distance from the inlet nozzle edge. It also appears advantageous to apply the nozzles of the diameters smaller than $2 \mathrm{~mm}$, and the measuring nozzle with narrower surface $D_{c} \leq 1.5$.

The measurement with digital devices proved to require the averaging of the obtained signal because of substantial fluctuations of the back-pressure. The averaging effectiveness is dependent highly on the localization of the measuring point. Assuming the maximal fluctuation of $\pm 50 \mathrm{~Pa}$ and proper localization of the probing point, it is possible to obtain a reliable signal every $0.2 \mathrm{~s}$.

\section{REFERENCES}

1. Bokov V.B. (2011), Pneumatic gauge steady-statemodelling by theoretical and empirical methods, Measurement, 44,303-311.

2. Breitinger R. (1971), New Pneumatic Measuring Gauges, Fertigungtechnik,61, 225-227, (in German)

3. Crnojevic C, Roy G, Bettahar A, Florent P. (1997), The Influence of the Regulator Diameter and Injection Nozzle Geometry on the Flow Structure in Pneumatic Dimensional Control Systems, Journal of Fluids Engineering, 119, 609-615.

4. Dejč M.E. (1961), Technical, Gosènergoizdat, Moskva-Leningrad, (in Russian)

5. Idel'čik I.E. (1960), Hydraulic Resistances Directory, Gosènergoizdat, Moskva, (in Russian)

6. Jermak Cz.J, Rucki M. (2016) Dynamics of the non-contac troundness measurement with air gages, Acta Mechanica et Automatica, 10(3),227-232.

7. Jermak Cz.J. (2010), Methods of Shaping the Metrological Characteristics of Air Gages, Journal of Mechanical Engineering, 56(6), 385390.
8. Jermak Cz.J. (2012), Theoretical and Practical Aspects of Shaping of Static Metrological Properties of Length Air Gauges Wyd. Politechniki Poznańskiej, Seria Rozprawy, nr 476, (in Polish)

9. Jermak Cz.J., Rucki M. (2012),Air Gauging: Static and Dynamic Characteristics, IFSA, Barcelona.

10. Kazimierski Z., Krysiński J. (1981), Gas Bearing Arrangement and Microturbine Drives, WNT, Warszawa, (in Polish)

11. Lammel L., Osiadacz A. (1974), Pneumatic Signals in Automation, WNT, Warszawa, (in Polish)

12. Lotze W. (1971), Design of Pneumatic Air Gauges, Feingerätetechnik, 29(9), 389-394, (in German)

13. MinaevA.N. (1987), Mechanics of Liquid and Gas, Metallurgiâ, Moskva, (in Russian)

14. Oleśkiewicz-Popiel Cz. (1981), Axial-symmetric Free and Striking Stream, Wyd. Politechniki Poznańskiej, Poznań. (in Polish)

15. Project Report (2001), Design of Metodology of Improving of Properties of Pneumatic Lenght-measurement Gauges. Project Report KBN 1253/T07/00/18. (in Polish)

16. Prosnak W.J. (1971), Fluid Mechanics - Gas Dynamics, t. 2, PWN, Warszawa, (in Polish)

17. Rucki M. (2009), Reduction of Uncertainty in Air Gauge Adjustment Process, IEEE Transactions on Instrumentation and Measurement, 58(1), 52-57.

18. Schuetz G. (2015), Pushing the Limits of Air Gaging-And Keeping Them There, Quality Magazine, 54(7),22-26.

19. Tanner C.J. (1958), Air gauging - history and future developments, Institution of Production Engineers Journal, 37(7),448-462.

20. Theory of Pneumatic Sensors Integrated with Piezoceramical Pressure Transducer (1986-1990), Annual Research Report.CPBP 02.20, $\mathrm{Nr}$ III.02, Poznań-Warszawa. (in Polish)

21. Tesar V. (2008), Characterisation of subsonic axisymmetric nozzles, Chemical Engineering Research and Design, 86,1253-1262.

22. Tesar V. (2010), Characterisation of inexpensive, simplyshaped nozzles, Chemical Engineering Research and Design, 88, 1433-1444.

23. Vacharanukul K., Mekid S. (2005), In-Process Dimensional Inspection Sensors, Measurement, 38(3),204-218.

24. Zalmanzon L.A. (1961), Flowing Elements of Pneumatic Control Appliances, Izd. Akademii Nauk SSSR, Moskva. (in Russian)

25. Zelczak A. (2002), Pneumatic Length Measurements, WKŁ, Warszawa, (in Polish)

Acknowledgement:The investigations had been performed in frames of the projects CPBP 02.20 and KBN 1253/T07/00/18 financed by Polish Government. 